Postgrad. Med. J. (1966), 42, 656.

Current Survey

\title{
PULMONARY EMBOLISM
}

\author{
M. PANeTh, F.R.C.S.
}

Brompton Hospital, London, S.W.3.

\begin{abstract}
The Incidence
CONFIDENCE in modern techniques of post-operative management and the increasing variety and magnitude of surgical interventions has caused an increased incidence of post-operative pulmonary embolism and infarction, but analysis of reported incidence indicates clearly that this is not the only factor; reports from countries devastated by the two world wars shows that the incidence falls significantly during and immediately after such a national disaster. This decrease in incidence can be related directly to the economic status of the population at risk, and is reversed when the nutritional state rises and, in particular, with the rise in the consumption of fats and proteins. There is also suggestive, but not conclusive evidence of a seasonal variation.

Morrell, Truelove and Barr (1963) showed that there was a five-fold rise in incidence from 1952 - 1961 in two hospitals under review, with a four-fold rise in mortality rate. In patients in medical wards, pulmonary embolism is an appreciable risk over the age of thirty, and the incidence rises sharply over the age of fifty; in surgical wards, the risk is very low below the age of forty, and rises less sharply after the age of fifty. In the two hospitals studied they found that a potentially preventable death occurred once every two weeks. Phear (1960) was able to collect and analyse 168 cases over a three-year period, from one hospital. His conclusions are important and will be referred to again.
\end{abstract}

\section{The Etiology}

Of all the factors of etiological significance in thrombo-embolism none appears more important than the limitation of normal activity imposed either by the patient's disease or by the restrictions of out-dated hospital discipline. The reduction of systemic venous flow, together with trauma doubtless forms the broad base upon which all thrombo-embolic phenomena depend. Failure of early post-operative ambulation to control embolism is probably largely due to the failure to recognise pre-operative thromboses. About half the patients who die after a variable period of confinement to bed demonstrate deep venous thromboses unsuspected in life. The majority of emboli originate in the intramuscular veins of the legs, and these veins enlarge with age. The physiological increase in platelet stickiness, which is a normal response to injury, must also be a factor, apart from such obvious ones as haemoconcentration and sludging in the micro-circulation due to dehydration and a reduced cardiac output.

\section{The Diagnosis}

As long ago as 1934 Churchill stated "the very fact that the event is so unexpected and tragic has coloured the observations with inaccuracies, and it is exceedingly difficult to find an adequate analysis of what really took place." In the major episode signs of infarction are very unusual, occuring only in $10 \%$ of cases, and signs of phlebitis are only found in $15 \%$ of cases. It has alsê been said that in cases operated on for pulmonary embolism the diagnosis has been wrong in more thar $60 \%$.

Massive pulmonary embolism may be regarded a causing acute obstruction of the right ventricle, leadinger rapidly to right ventricular paralysis, and this is reflecteos in the survival times. Gorham, in a retrospective study of 100 fatal cases of proven pulmonary embolism found that 41 patients died within ten minutes of the major episode, three more within 15 minutes, but 220 survived two hours and 34 survived up to two weeks This analysis was made before the universal acceptancer of external cardiac massage, and it is not unreasonable to presume that more than $50 \%$ of patients will in future survive the initial onslaught long enough for moderm techniques to be mobilised effectively for successfuR embolectomy.

If logical and deductive reasoning is applied to establishing the diagnosis, then it is not a difficult to make, and the margin for error beccmes small can be eliminated by special investigations in those cases in which the diagnosis remains in doubt on clinimg grounds. An embolus large enough to endanger lifes does so by:

(1) Obstructing more than $70 \%$ of the pulmonary arterial tree and,

(2) Reducing the cardiac output.

The clinical features are those of acute right hear $\overrightarrow{\vec{B}}$ failure, coupled with a sudden fall in cardiac output? The patient, with or without a classical preceding history? of an operation ten days or more before, a call to stool? retrosternal pain or discomfort followed by syncope will present with:

(1) A raised central venous pressure,

(2) Tachypnoea,

(3) Sinus tachycardia,

(4) Cold extremities with peripheral cyanosis,

(5) A low systolic blood pressure, and

(6) A right ventricular gallop;

if all these clinical signs are present then the diagnosis of acute, massive right ventricular obstruction cannot: be denied. It has been stated that there may be,

(1) A loud pulmonary component of the heart sound at the base. This is only observed in chronic obstruction 0 to the right ventricle where the ventricle has hypertrophied to adjust to the increased pulmonary vascular resistance ${ }_{5}$ and is therefore never heard in the acute case because the normal right ventricle is incapable of generating a high pressure for any length of time. The presence of a loud pulmonary second sound should therefore put one on one's guard.

(2) A murmur. By the same argument, in the acute case the right ventricle is incapable of producing an sufficiently forceful ejection of blood past the partialo 
obstruction to cause a murmur and, also, the reduction in cardiac output prevents the generation of a murmur.

(3) Pulsation in the second intercostal space due to a distended pulmonary artery. The pulmonary artery is not distended in these cases, for reasons similar to (1) and (2).

The differential diagnosis rests between coronary infarction, dissecting thoracic aneurysm, cardiac tamponade, massive post-operative mediastinal haematoma and tension pneumothorax. In coronary infarction, the pain is more severe and persistent, and the systemic venous pressure is not so markedly raised unless heart failure is present. Rhythm changes are also common, whereas they are uncommon in pulmonary embolism. The ECG will often confirm the diagnosis. Dissecting thoracic aneurysm usually affects the peripheral pulses unequally, and, classically, produces typical pain and widening of the mediastinum on the chest X-ray. Cardiac tamponade is one of the more difficult differential diagnoses, but can be distinguished by the systolic descent of the venous pressure in the neck. There will also be an arterial pulsus paradoxus, which should be easy to elicit if the tamponade is severe enough to lower the systemic output to critical levels. Mediastinal haematoma can usually be seen on the chest X-ray, and a pneumothorax can be distinguished clinically and also on the chest X-ray.

\section{Special Investigations}

The ECG will show the changes of acute right ventricular strain, that is to say a dominant $S$ in lead 1 , $T$ inversion and $Q$ waves in lead 3, and $T$ wave inversion in V1 - 4. RBBB may also occur. Serial ECGs, if available, will show a progressive swing of the electrical axis to the right. (Towers, 1966).

The X-ray is normal in most cases unless the massive episode has been preceded by a matter of days by a peripheral pulmonary infarct, when lobar or segmental consolidations, with or without effusions, may be present.

In $80 \%$ of cases the diagnosis is based on the clinical signs, the ECG and the chest X-ray. In cases of doubt the most useful other investigation is the Pulmonary Arteriogram, but this should only be performed if doubt exists as to the diagnosis or the degree of obstruction. It should only be carried out in a department of angiography, and in critically ill patients it carries a risk, but Williams and Wilcox, in 1963, described a simple technique in a series of 50 cases, half of which were acute obstructions, and obtained positive angiographic evidence of major block in $75 \%$. One would stress that in the critically ill patient the diagnosis is not in doubt on clinical, ECG and plain chest X-ray evidence, and the so-called mild case can be transferred, if necessary, to a special centre, with special facilities for investigation. Radioactive scanning of the chest, after injection of radioactive aggregated serum albumen, is neat but requires expensive equipment and is not yet a widely accepted investigation.

\section{The Treatment}

Prevention by maintenance of venous flow, as by early ambulation and leg exercises, has not been as successful as it was hoped. The routine use of anticoagulants post-operatively, increasing the prothrombin time to twice normal, significantly affects the incidence of pulmonary embolism and infarction. In itself, this form of treatment is not without its own risks as the prothrombin time may be difficult to control in the immediate post-operative period. Ligation of veins and plication of the inferior vena cava in established phlebothrombosis carries its own morbidity; and the indications are not so clearly defined as to make this an attractive measure (Donaldson, Williams, Scannell and Shaw, 1961). The simplest measure is vigilance on the part of all resident staff and prompt and effective heparinisation at the first sign of phlebothrombosis, or the warning pulmonary infarct. It should be realised that heparinisation does not prevent subsequent major embolism in all cases.

\section{Indications for Embolectomy}

Once the diagnosis is made the indications may be listed as follows :-

(1) One or more attacks of cardiac arrest.

(2) A systemic blood pressure at or below $100 \mathrm{~mm}$. $\mathrm{Hg}$ systolic.

(3) A falling cardiac output.

(4) Tachypnoea associated with arterial desaturation; this is indicative of a gross perfusion/ventilation imbalance.

(5) Evidence of repeated pulmonary embolism.

Surgical technique: Except when circumstances make it unavoidable, the operation should be performed witho the aid of cardio-pulmonary bypass. The ready avail ability of sterile, mass-produced bubble oxygenators has made this operation a safe emergency procedure? (Cooley, Beall, and Alexander, 1961). Since the cardiac output is fixed at a low level, measures to prevent the vaso-dilatation occurring on induction of anaesthesia are life-saving. Supportive femero-femoral perfusion of a limited minute volume is attractive and logical in theory, but requires skill and experience in practice. Metaraminol (Aramine) intravenously effectively counteracts the fatal fall in blood pressure and allows full bypass to be established by,

(i) a vertical median stenotomy, the pericardium also being opened vertically;

(ii) rapid cannulation of the right atrial appendage for gravity venous drainage into the oxygenator; and

(iii) cannulation of the ascending aorta for arterial return.

Once bypass is established, the heart is electively fibrillated and both pulmonary arteries are carefully, and meticulously cleared of all thrombi down to their basal divisions, through a longitudinal incision in the pulmonary trunk extending up to its bifurcation. Milking of both lungs towards their hila is advocated by some (Cooley and colleagues, 1961) but may not be necessary in every case. With the pleural sacs intact, inflation of the lungs by the anaesthetist will flush back emboli if the pulmonary trunk is open.

In properly managed cases, the operation is simple, easy and quick. Early reports of the results of this procedure are encouraging; the mortality arises chiefly from delay in diagnosis and treatment. Causes of death in most published series are due to cerebral damage, 
irreversible right ventricular failure and renal failure, the result of a low cardiac output pre-operatively.

The answer to the question of re-canalisation of the pulmonary arteries is not yet satisfactory. Although Allison, Dunhill and Marshall in 1960 have shown that experimentally produced autologous pulmonary emboli disappear in four weeks, this is not invariably so in clinical practice. Phear's analysis of the long-term follow up of survivors treated medically clearly indicates that rhythm changes, cor pulmonale and respiratory insufficiency occurs in the older age group.

There is therefore a further indication for embolectomy: the prevention of these late complications, although the obstruction may not be severe enough to endanger life immediately.

Trendelenburg, in his classical paper in 1908, stated "Two conditions are necessary for successful embolectomy; first, the diagnosis must be right and, second, there must be enough time to remove the obstruction," and he concludes his paper by stating "This much we have learned from our cases, that the diagnosis in all surgical cases is easy; invariably did we find the emboli." The diagnosis is straightforward if the basic principles underlying it are borne in mind. Time can be bought by effective vasopressors and modern surgical technique using cardio-pulmonary bypass.

\section{REFERENCES}

Allison, P. R., Dunhill, M. S., and Marshall, R.̊ (1960): Pulmonary Embolism, Thorax, 15, 273.

Cooley, D. A., Beall, A. C., and Alexander, J. K. (1961): Acute Massive Pulmonary Embolism. Successful Surgical Treatment using Temporary Cardiopulmonary Bypass, J. Amer. med. Ass., 177, 283.

Donaldson, G. A., Williams, C., Scannell, J. G., and SHAw, R. S. (1963): A Reappraisal of the Application $\frac{\Gamma}{\sqrt{2}}$ cf the Trendelenburg Operation to Massive Fatal $\mathbb{Q}$ Embolism. Report of a Successful Pulmonary-artery Thrombectomy using a Cardiopulmonary Bypass, $\infty$ New Engl. J. Med., 268, 171.

Gorham, L. W. (1961): A Study of Pulmonary Embolism. Part I. A Clinicopathological Investigation of 100 Cases of Massive Embolism of the Pulmonary Artery; ${ }_{\sigma}$ Diagnosis by Physical Signs and Differentiation fromo Acute Myocardial Infarction, Arch. intern. Med., 108, 8.0

Morrell, M. T., Truelove, S. C., and Barr, A. (1963) Pulmonary Embolism, Brit. med. J., ii, 830.

PHEAR, D. (1960): Pulmonary Embolism. A Study of Late Prognosis, Lancet, ii, 832.

Towers, M. K. (1966): Chronic Cor Pulmonale, Postgrad.N med. J. 42, 506.

TRENDELENBURG, F. (1908): Uber Die Operative Be-ת̆ handlung Der Embolie Der Lungenarterie, Arch. klin, 으 Chir., 86, 686.

Williams, J. R., and Wilcox, W. C. (1963): Pulmonary Embolism. Roentgenographic and Angiographic Consideration, Amer. J. Rcentgenol., 89, 333. 